\title{
A new model for dermatitis herpetiformis that uses HLA-DQ8 transgenic NOD mice
}

\author{
Eric Marietta, ${ }^{1}$ Kay Black, ${ }^{1}$ Michael Camilleri, ${ }^{2}$ Patricia Krause, ${ }^{3}$ Roy S. Rogers III, ${ }^{3}$ \\ Chella David, ${ }^{1}$ Mark R. Pittelkow, ${ }^{3}$ and Joseph A. Murray ${ }^{4}$
}

1Department of Immunology, Mayo Clinic College of Medicine, Rochester, Minnesota, USA. 2Department of Dermatology, Sir Paul Boffa Hospital, Floriana, Malta. ${ }^{3}$ Department of Dermatology, and ${ }^{4}$ Department of Internal Medicine, Mayo Clinic College of Medicine, Rochester, Minnesota, USA.

\begin{abstract}
Dermatitis herpetiformis (DH) is an autoimmune blistering skin disorder that is associated with gluten sensitivity. It presents as a papulovesicular rash and is often associated with enteropathy. The rash resolves when the patient is placed on a gluten-free diet and/or dapsone. DH, as well as celiac disease, is tightly associated with DQ2 and DQ8. A novel mouse model for DH is described that utilizes the NOD background and the HLA-DQ8 transgene. The addition of DQ8 contributes sensitivity to gliadin, and the addition of the NOD background contributes to autoimmunity and pathogenesis. Fifteen NOD $\mathrm{DQ8}^{+}$mice of 90 that were sensitized to gluten developed blistering pathology similar to that seen in DH. Neutrophil infiltration of the dermis, deposition of IgA at the dermal-epidermal junction, and a complete reversal of the blistering phenomenon with the administration of a gluten-free diet with or without dapsone were observed. None of the 3 blistering mice examined had small-bowel pathology. This animal model of DH will be useful to determine the specificity of the IgA deposits, as well as the pathogenic mechanisms that occur in the skin as a result of gluten ingestion.
\end{abstract}

\section{Introduction}

Dermatitis herpetiformis (DH) is a chronic skin disorder that was first identified by Duhring in 1884 (1). It is an autoimmune, blistering, cutaneous condition that presents with an intensely pruritic papulovesicular rash on the elbows, forearms, buttocks, knees, and scalp (2). DH is characterized histologically by subepidermal blisters with an upper- and mid-dermal mixed inflammatory infiltrate. The predominant inflammatory cell composing the infiltrate is the neutrophil, with prominent eosinophil infiltration occurring in $25 \%$ of cases $(1,3,4)$. A pathognomonic finding in $\mathrm{DH}$ is the presence of granular deposits of IgA along the basement membrane zone and at the tips of the dermal papillae of perilesional uninvolved areas of the skin. The presence of these IgA deposits is detected by direct immunofluorescence analysis. $\mathrm{DH}$ is often associated with a gluten-sensitive enteropathy marked by villous atrophy and increased infiltration of intraepithelial lymphocytes $(3,5)$. Both the rash and the enteropathy resolve upon the withdrawal of gluten from the diet (6). DH and celiac disease are initiated by the ingestion of wheat and related grains containing gluten. Both diseases are strongly associated with HLA-DQ2 and HLA-DQ8, such that $86 \%$ of all DH patients and celiac patients are $\mathrm{DQ}^{+}$and most of the remainder are $\mathrm{DQ}^{+}$ (7). Reports of monozygotic twin pairs that were discordant for $\mathrm{DH}$ and celiac disease indicate that the environment contributes to the development of $\mathrm{DH}$ or celiac disease in those individuals that are both genetically susceptible and identical $(6,8)$. For both diseases, a gluten-free diet resolves the symptoms; however, $\mathrm{DH}$ also responds to the antineutrophilic drug dapsone $(9,10)$. The administration of dapsone accelerates resolution of the blistering

Nonstandard abbreviations used: $\mathrm{Ab}^{\circ}$, lacking endogenous mouse MHC II; ACD, allergic contact dermatitis; cpdm, chronic proliferative dermatitis; $\mathrm{DH}$, dermatitis herpetiformis; fsn, flaky skin; Idd, insulin-dependent diabetes; TGe, epidermal transglutaminase; tTG, tissue transglutaminase.

Conflict of interest: The authors have declared that no conflict of interest exists.

Citation for this article: J. Clin. Invest. 114:1090-1097 (2004).

doi:10.1172/JCI200421055 in $\mathrm{DH}$ compared with a gluten-free diet alone (4) and does not alter the gastrointestinal pathology seen in DH patients (11).

Multiple mouse models of atopic dermatitis and other inflammatory skin disorders have been developed (12). These include the $\mathrm{NC} / \mathrm{Nga}$ mouse (13), the chronic proliferative dermatitis (cpdm) mouse (14), the flaky skin (fsn) mouse (15), the DS-Nh mouse (a DS mutant strain that is deficient in hair growth) (16), the allergic contact dermatitis (ACD) mouse (17), and a mouse model of atopic dermatitis associated with food hypersensitivity (18). The NC/Nga model is a model for atopic dermatitis and is marked by an overproduction of $\mathrm{IgE}$ in conventional colonies. The cpdm model is a spontaneous mutation in C57BL/KaLawRij mice and is similar to chronic dermatitis found in humans. The dermatitis in these mice is generated by the infiltration of mast cells, macrophages, and eosinophils, which is different from the process of $\mathrm{DH}$ (19). fsn is a spontaneous mutation in mice that is similar to human psoriasis and has been used as a model of hyperproliferative inflammatory alteration of the skin, which also is distinct from DH. Dermatitis has been induced in the ACD mouse by topical application of 2.4-dinitrofluorobenzene (DNFB) and FITC. The mouse model of atopic dermatitis associated with food hypersensitivity is notable for hypersensitivity generated against cow's milk or peanuts, similar to the IgE-mediated peanut hypersensitivity observed in humans. These models contributed significantly to our understanding of immune-inflammatory mechanisms that mediate dermatitis.

Recently, a passive-transfer mouse model of IgA autoimmune disorders was developed (20). This particular model used athymic mice or SCID mice as recipients of human skin grafts. For linear IgA bullous dermatosis (LABD), purified IgA or IgG anti-human LABD97 was injected into the mice. Both IgA and IgG were observed to deposit at the basement membrane zone. By contrast, sera from DH patients were injected into athymic mice that had received human skin grafts, but these did not yield the granular IgA deposits at the basement membrane zone that are found in $\mathrm{DH}$ patients.

Spontaneous or genetically modified animal models that most closely approximate gluten sensitivity are Irish setters that develop 


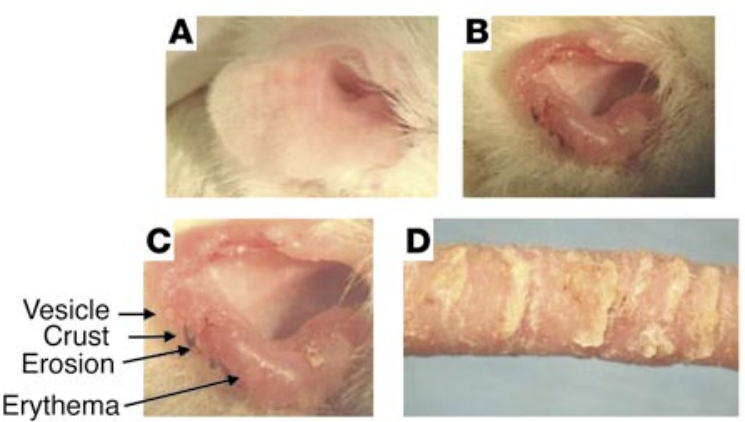

Figure 1

Progression of cutaneous blistering. (A) Normal unaffected ear. (B) Blistered ear of an NOD Ab ${ }^{\circ} \mathrm{DQ8}^{+}$mouse sensitized to gluten. (C) Magnified view of the ear in B. (D) Tail of an affected mouse.

sensitivity to wheat (21) and HLA-DQ8 ${ }^{+}$transgenic mice (22). The Irish setter model is promising because of the wheat-associated enteropathy but lacks the other components of celiac disease, such as defined genetic risk. There also has not been a report of blistering pathology in the Irish setter model. The DQ8 transgenic mouse model carried a genetic component that contributed to gluten sensitivity (DQ8) but lacked gluten-sensitive enteropathy or blistering. These $\mathrm{DQ}^{+}$mice also failed to develop antibodies against tissue transglutaminase (tTG; type II transglutaminase), which represents part of the autoimmune component observed in celiac disease (22).

In order to improve upon the transgenic DQ8 ${ }^{+}$mouse model, we incorporated 2 new components. The first component was a predisposition toward autoimmune disease that was introduced by backcrossing of $\mathrm{DQ}^{+}$mice lacking endogenous mouse $\mathrm{MHC}$ II $\left(\mathrm{Ab}^{\circ} \mathrm{DQ}^{+}\right.$mice) with NOD mice (a classical murine model for type 1 diabetes and other autoimmune diseases) to generate congenic NOD Ab ${ }^{\circ} \mathrm{DQ}^{+}$mice (23). The second component was an inflammatory trigger to initiate disease. We chose pertussis toxin for its adjuvant properties and utility in generating autoimmune diseases $(24,25)$.

Unexpectedly, when these novel congenic NOD $\mathrm{Ab}^{\circ} \mathrm{DQ}^{+}$mice were being sensitized to gluten and simultaneously injected with pertussis toxin, some developed blisters and other skin abnormalities on their ears. Histologic and direct immunofluorescence analysis demonstrated that these mice were experiencing subepidermal blister formation composed of neutrophils, monocytes, and occasional eosinophils in the upper dermis in conjunction with granular IgA deposits in the region of the basement membrane zone, which is pathologically consistent with DH. Induction of blisters by the ingestion of gluten was also observed. Thus, these mice manifest the 3 features that are used clinically to diagnose DH: gluten-dependent blistering, granular IgA deposition localizing beneath the basement membrane zone, and subepidermal blisters rich in neutrophils and monocytes within the dermis. The present series of studies reports the major similarities and differences observed between $\mathrm{DH}$ and this gluten-sensitive NOD $\mathrm{Ab}^{\circ}$ $\mathrm{DQ}^{+}$transgenic mouse model of $\mathrm{DH}$ and discusses the specificity of the IgA that is deposited at the dermal-epidermal junction.

\section{Results}

Development of gluten-dependent blistering. Fifteen of 90 glutensensitized $\mathrm{NOD} \mathrm{Ab}^{\circ} \mathrm{DQ} 8^{+}$mice developed blisters on both ears after 2-5 months of gluten ingestion. They appeared as translu- cent to whitish papulovesicles, which ruptured and broke down into erythematous erosions that scabbed. Mice were routinely observed scratching their ears during this period. With more extensive blistering over time, diffuse erythema and thickening of the ear occurred, such that the architecture of the ear began to change and the normal shape involuted. Figure 1 displays the progression of blistering from the initial stage to the involuted stage, which occurred over approximately 6 weeks. Figure 1A depicts a normal unaffected ear. Figure 1, B and C, displays an affected ear with cream-colored papulovesicles, erosions, scaling of the ear, crust formation, erythema, thickening, and slowly progressive involution of ear tissue. Figure 1D displays the tail of one of the blistering mice. Note that scales have formed. Histologic analysis of the tail showed that it was colonized with bacteria, which was probably due to exposure of the heavily blistered areas to the feces at the bottom of the cage. Interestingly, the ears were the primary site for blistering. On some mice, the tail, the sides of the muzzle, and periocular skin were affected, as well as the sides of the paws. However, ear involvement was a consistent finding, and the blistering of the paws, tail, muzzle, and eyes was coincident with involvement in the ears. No blistering was observed over the stomach or back skin, where a dense coat was present.

The affected tissue also appeared to be sensitive for the mice, since they would often scratch their ears after being touched. This behavior mimics that of human patients with DH, who typically experience marked pruritus of involved skin sites.

Histopathology of the ear. The H\&E-stained lesional skin specimens revealed subepidermal blisters with upper- to mid-dermal mixed inflammatory infiltrates composed of neutrophils, eosinophils, lymphocytes, and histiocytes, as seen in Figure 2. Biopsies were performed on all 15 blistered mice. These findings are similar to those seen in lesional skin of human DH patients.

Cellular infiltration of the lesional skin. Direct immunofluorescence analysis of the lesional tissue revealed that the epidermis stained positively for the $7 / 4$ neutrophil marker, as compared with the isotype control (Figure 3, B and C). Neutrophil elastase localized to the epidermis as well as to polymorphonuclear leuko-

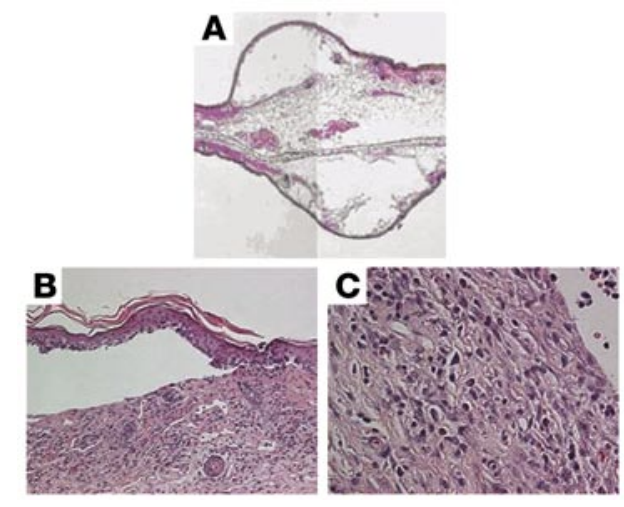

\section{Figure 2}

Histopathology of a blistered ear. H\&E-stained sections from the lesional areas of blistered ears from gluten-sensitized NOD Ab ${ }^{\circ} \mathrm{DQ}^{+}$ mice. (A) Composite image of subepidermal blistered ear. Original magnification, $\times 5$. (B) Full-thickness epidermal separation from the dermis of a gluten-sensitized NOD Ab ${ }^{\circ} \mathrm{DQ}^{+}$mouse. Original magnification, $\times 20$. (C) Mid-dermal infiltration with localized dermal edema of mixed inflammatory cells. Original magnification, $\times 40$. 


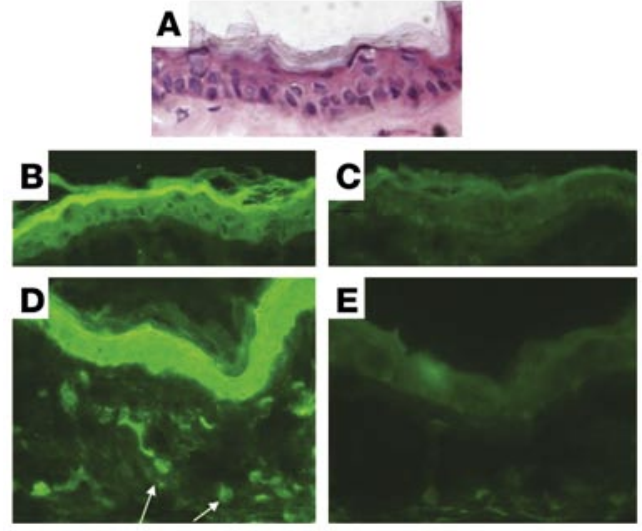

Figure 3

Direct immunofluorescence analysis of the cellular infiltration of lesional skin. (A-E) Lesional sections of the ear of an NOD Ab ${ }^{\circ} \mathrm{DQ}^{+}$ mouse that had developed gluten-dependent blisters. (A) H\&E staining. Original magnification, $\times 20$. (B) Anti-7/4 staining of epidermis from a lesional mouse. Original magnification, $\times 20$. (C) Isotype control. Original magnification, $\times 20$. (D) Anti-neutrophil elastase staining of the epidermis and upper dermis of a lesional mouse. Original magnification, $\times 20$. (E) Isotype control. Original magnification, $\times 20$. Arrows in the upper dermis identify elastase-containing neutrophils.

cytes within the dermis of blistering mice as compared with the isotype control (Figure 3, D and E). No positivity was seen in the ears of naive mice (data not shown). Neutrophil elastase was also present adjacent to the regions of separation between the epidermis and the dermal layer (data not shown). No $\mathrm{CD}^{+}$or $\mathrm{CD}^{+}$ cells were detected in the lesional biopsies of affected mice (data not shown). Staining with anti-c-kit and anti-MBP antibodies revealed small numbers of reactive cells present throughout the ear tissue of both gluten-sensitized and naive $\mathrm{NOD} \mathrm{Ab}^{\circ} \mathrm{DQ}^{+}$ mice (data not shown). Therefore, no significant alteration in the number, or localization, of mast cells or eosinophils within the lesional areas occurred in the ears.

Deposition of IgA. Direct immunofluorescence examination of the perilesional uninvolved skin revealed granular deposits of IgA encroaching on the basement membrane zone (Figure $4, A, C$, and D), as well as at the tips of the dermal papillae (Figure 4B), similar to biopsies of perilesional, nonblistered skin from DH patients (26). In Figure 4, D-F, the IgA deposits lie below the basement membrane zone within the upper dermis as defined by collagen IV staining. IgG deposits were not detectable along the basement membrane zone or in the papillary dermis of any of the specimens. Ear tis-

\section{Figure 4}

IgA dermal deposit localization. (A) FITC staining identifies IgA deposition in perilesional uninvolved areas of NOD Ab० ${ }^{\circ} 8^{+}$mice with gluten-dependent blistering. Original magnification, $\times 20$. (B) Deposition of IgA at the tips of papillae. Original magnification, $\times 20$. (C) Granularity of the IgA deposits below the basement membrane zone. Original magnification, $\times 100$. (D) FITC staining identifies $\lg A$. Original magnification, $\times 100$. (E) Rhodamine Red-X staining identifies collagen IV. Original magnification, $\times 100$. (F) Composite picture with both IgA staining and collagen IV staining. Original magnification, $\times 100$. Arrows identify IgA deposits, and the basement membrane zone is highlighted by a dotted white line. sue of naive $\mathrm{NOD} \mathrm{Ab}^{\circ} \mathrm{DQ}^{+}$mice did not harbor any granular deposits along the dermal-epidermal junction (data not shown). Also, no IgA deposits were found in the back skin of blistering mice, where no blisters were observed to form (data not shown). Similar negative findings have been recorded in the lesion-free, blister-resistant skin of DH patients (26). However, others have observed decreased amounts of IgA deposits in uninvolved skin of DH patients $(27,28)$. Biopsies were also performed on blistered (infected) tails, and no IgA deposits were found. IgA deposits were not observed in the lesional skin of blistering mice, and none of 8 nonblistering mice that had been gluten-sensitized and given a high-gluten diet for between 2 and 4 months had developed IgA deposits.

Gluten dependence of the blistering. To determine whether the blistering phenomenon observed in the HLA-DQ8 transgenic NOD mouse was dependent on the ingestion of gluten, 9 gluten-sensitized mice that had developed blisters were placed on a gluten-free diet with or without dapsone treatment and monitored for further blistering. Resolution of the blisters occurred within 1 week after 3 mice were given a gluten-free diet and treated with dapsone. Six mice were treated with a gluten-free diet alone (no dapsone), and their blisters began to resolve after 2-3 weeks. Complete resolution of blistering depended on the extent of the blistering when the mouse was placed on the gluten-free diet alone, such that minor blistering was resolved after the second week of gluten-free diet and extensive blistering required greater than 2 weeks of glutenfree diet for blister resolution.

An example of this response is shown in Figure 5. Figure 5A displays the left ear of a gluten-sensitized NOD $\mathrm{Ab}^{\circ} \mathrm{DQ}^{+}$mouse that had developed papulovesicles at the tip of the ear. Figure 5B displays the same left ear after 5 weeks of a gluten-free diet and dapsone treatment. At this point, no new blisters had formed, and the architecture of the ear was normal with the exception of the region that had been removed for histology at the beginning of the gluten-free diet and dapsone treatment. This is in contrast to the ear of Figure 1B, which had extensive blistering and had lost its architecture after 5 weeks without a gluten-free diet and dapsone treatment. When Figures 1 and 5 are compared, it
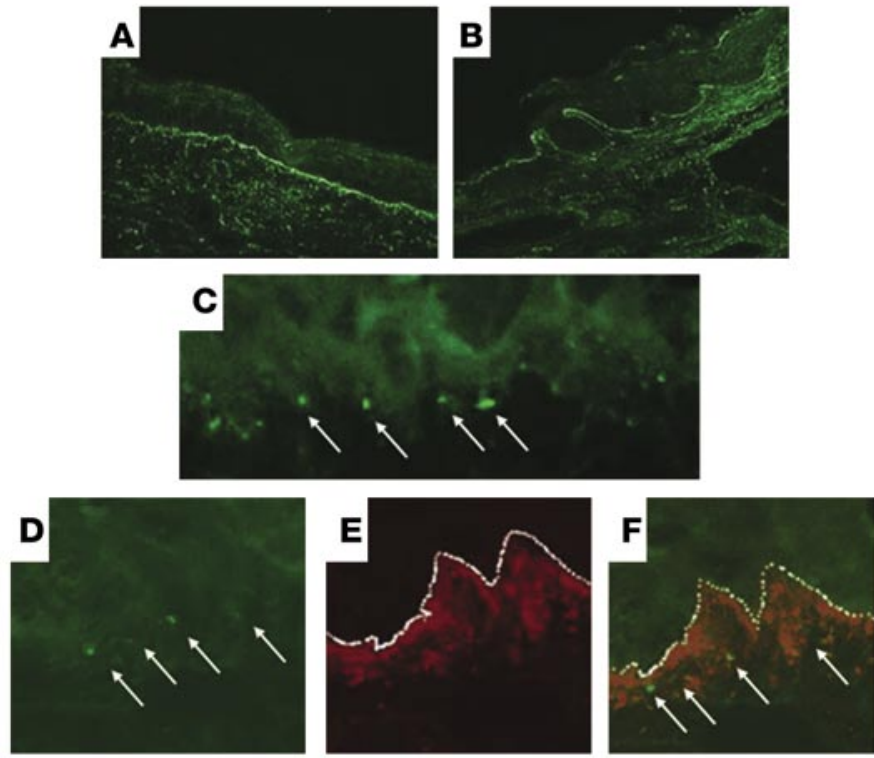

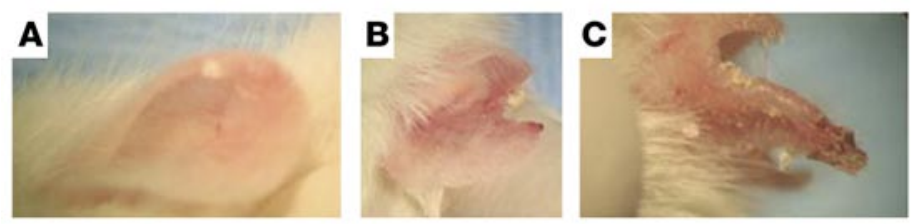

Figure 5

Gluten dependence. (A) Ear of a gluten-sensitized NOD Ab DQ8+ mouse in the initial stage of blistering. (B) The ear in $\mathbf{A}$ after 5 weeks of gluten-free diet and dapsone treatment. (C) The same ear after gluten rechallenge. Biopsies were taken before and after dapsone treatment.

is clear that the combination of a gluten-free diet and dapsone inhibits the formation of new blisters and ear involution. The dependence on gluten becomes even more apparent as evidenced in Figure 5C, which shows the same left ear as in Figure 5, A and B. The extensive blistering with erythema and thickening in this ear is the result of discontinuation of gluten-free diet and dapsone treatment and rechallenge with gluten for 5 weeks. Of note, perceptible blisters redeveloped 1 week after gluten rechallenge for 2 mice that were rechallenged with gluten after a gluten-free diet and dapsone treatment, as well as a mouse that was only treated with a gluten-free diet.

This finding demonstrates that the blistering phenomenon is a direct consequence of reingestion of gluten once the mice have been sensitized to gluten. Interestingly, in contrast to the 2- to 5 -month period of gluten ingestion required after gluten sensitization for the initial appearance of blisters, perceptible blisters redevelop within approximately 1 week after gluten rechallenge, indicating a memory immune response to gluten. The disappearance of blisters with a gluten-free diet with or without dapsone treatment and the reappearance of blisters with gluten rechallenge, together, demonstrate that the blistering phenomenon is dependent on the ingestion of gluten. The responses of these mice to gluten challenge, gluten withdrawal with or without dapsone, and gluten rechallenge are similar to the lesions of human DH patients.

Onset and disease susceptibility. The onset of disease was observed to develop between 2 and 5 months after gluten injection and a gluten enriched diet. Thus far, no mice have developed initial blistering past 5 months after injection, with some mice observed as long as 8 months after injection. No blistering or IgA deposition was observed in $\mathrm{NOD}, \mathrm{NOD} \mathrm{Ab}^{\circ}$, or $\mathrm{Ab}^{\circ} \mathrm{DQ}^{+}$ mice after gluten sensitization, which indicates that both the DQ8 transgene and the NOD background are required in combination for the development of gluten-dependent blistering with IgA deposition; yet individually, each is insufficient to induce the skin disease.

Incidence of DH-like symptoms. Fifteen of $90 \mathrm{NOD} \mathrm{Ab}{ }^{\circ} \mathrm{DQ}^{+}$ mice that were sensitized to gluten developed blisters. No blistering was observed in NOD and NOD $\mathrm{Ab}^{\circ}$ control mice that were treated similarly. Blistering was not observed in NOD $\mathrm{Ab}^{\circ} \mathrm{DQ}^{+}$ mice that were alternatively injected with pertussis toxin alone, injected with gluten alone, given only gluten gavage, injected with pertussis toxin and gluten without gluten gavage, or injected only with pertussis toxin and given gluten gavage. IgA deposits were detected in the perilesional uninvolved skin of 11 of the 15 blistering mice. No IgG deposits were found in any of the 15 blistering mice. Nine of the 15 blistering mice were treated with a gluten- free diet with or without dapsone 1 month after the first presentation of blisters, and their blisters and skin inflammation resolved. The 3 mice that were treated with a gluten-free diet and dapsone together had blister resolution within a week, whereas the 6 that were treated with a gluten-free diet alone took 2-3 weeks for the resolution of blisters to occur. Two mice treated with a gluten-free diet and dapsone and 1 mouse treated with only a gluten-free diet were later rechallenged with gluten and were observed to redevelop blistering in 1 week, as depicted in Figure 5.

Interestingly, 1 blistering mouse that had been treated with a gluten-free diet and dapsone for 5 weeks, then rechallenged with gluten for 5 weeks, did not develop histologic evidence of enteropathy as determined by H\&E staining. No shortening of the villi or thickening of the lamina propria was observed in the ileum, jejunum, or duodenum, nor was there an increase in the number of intraepithelial lymphocytes in any of those 3 regions. Similarly, 2 severely blistering mice maintained for 5 months on the normal mouse chow that contained wheat and challenged biweekly with gluten also did not demonstrate any signs of enteropathy. The other 12 blistering mice were not examined for enteropathy. Thus, none of 3 blistering mice examined had small-bowel pathology. The blistering mice were not diabetic; the glucose levels in these mice were normal, varying between 60 and $100 \mathrm{~g} / \mathrm{dl}$.

Specificity of the antisera. To determine the possible antigen specificity of the IgA deposits, the sera of the blistering mice were tested. There were no IgA or IgG endomysial antibodies or IgA antibodies against guinea pig tTG detected in the sera of these mice, despite strong amino acid sequence similarity of approximately $80 \%$ between guinea pig and mouse tTG (29). No gliadin- or gluten-specific IgA was detected in the sera; however, gluten-specific IgG antibodies were found in the sera of the blistering mice. The sera from 2 blistered mice did not react with normal mouse skin, salt-split skin of a normal mouse, nor normal human salt-split skin.

Sardy et al. reported in 2002 that IgA of DH patients specifically reacted to epidermal transglutaminase (TGe; type III trans-

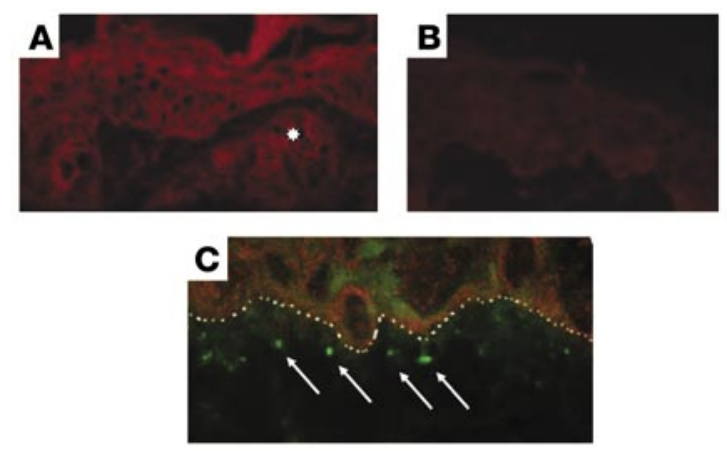

\section{Figure 6}

IgA deposits and TGe localization. (A) Expression of mouse TGe in a perilesional uninvolved section from the ear of a blistering mouse. The star identifies a follicular appendage. Original magnification, $\times 40$. (B) Isotype control to anti-TGe antibody. Original magnification, $\times 40$. (C) IgA deposition detected with FITC staining and TGe detected with Rhodamine Red-X. The dermal-epidermal membrane zone is highlighted by a dotted white line, and arrows identify IgA deposits. Original magnification, $\times 100$. 
glutaminase) (30). TGe-specific IgA was detected in the sera of none of 3 blistering mice, compared with 2 naive $\mathrm{NOD} \mathrm{Ab}^{\circ}$ $\mathrm{DQ}^{+}$mice (data not shown). The amino acid sequence similarity between mouse and human TGe is $84 \%$ and would not explain the absence of reactivity (31). Figure $6 \mathrm{C}$ shows a double stain that demonstrates the presence of mouse TGe and the location of the IgA deposits. As can be seen, TGe is expressed throughout the epidermis, and the IgA deposits are located below the dermal-epidermal interface.

\section{Discussion}

We report herein a novel study examining the pathology of a blistering disease observed in mice that is strikingly similar to pathology observed in the human skin disorder dermatitis herpetiformis. To our knowledge, this represents the first reported HLA transgenic mouse model of DH. The gluten-sensitized $\mathrm{NOD} \mathrm{Ab}^{\circ} \mathrm{DQ}^{+}$mice that developed blisters had granular IgA deposits along the basement membrane zone, had subepidermal blisters rich in neutrophils and monocytes, responded to a gluten-free diet with or without dapsone treatment, and redeveloped blisters with gluten rechallenge.

Despite the many similarities between this blistering disease and $\mathrm{DH}$, there are some differences, including the lack of enteropathy and of circulating antibodies against endomysium and tTG in the mouse disease. However, the inability to detect these antibodies or any enteropathy in the mice that we screened does not diminish more striking immunopathologic similarities to $\mathrm{DH}$, which include DQ8 dependence, gluten restriction, and IgA deposition beneath the lamina densa. We have therefore determined this blistering disease to be compatible with $\mathrm{DH}$.

A number of factors contribute to the development of blistering found in this mouse model. The first is expression of HLA-DQ8. This MHC molecule determines gluten sensitivity, as described by Black et al. (22). The second factor is the introduction of the NOD background. NOD has been well documented to contribute to the development of autoimmunity, especially diabetes. When this project was initiated, inclusion of the NOD background was intended to generate an autoimmune gluten-associated enteropathy. However, the blistering disease exclusively developed. These mice did not develop diabetes mellitus, since the glucose remained in the normal range. Presently, it is unclear what aspects of the NOD background contribute to the development of the immune blistering pathology. However, it is clear that the $\mathrm{NOD}$ background is necessary, since $\mathrm{Ab}^{\circ} \mathrm{DQ}^{+}$mice (non-NOD background) fail to develop the blistering disease after the same gluten-sensitization protocol (22). The contribution of a genetic background predisposing to autoimmunity is consistent with the known predisposition of $\mathrm{DH}$ patients to other autoimmunemediated diseases such as thyroiditis (32).

Identifying the genetic components contributed by the NOD background would be a major undertaking, but it is likely that the contributing factor(s) would be 1 or more of the greater than 19 loci that contribute to the development of spontaneous insulin-dependent diabetes (Idd) in the NOD mouse, called the Idd susceptibility loci. The NOD mice do not develop the glutendependent blistering disease, in contrast to the NOD $\mathrm{Ab}^{\circ} \mathrm{DQ}^{+}$ mice that were generated for this model. The NOD Ab ${ }^{\circ} \mathrm{DQ}^{+}$ mice lack some Idd loci mapping to the 17 th chromosome, as it was replaced with the chromosome containing the $\mathrm{Ab}^{\circ}$ genes. However, the NOD $\mathrm{Ab}^{\circ} \mathrm{DQ}^{+}$mice express most of the NOD Idd loci. In comparing the types of autoimmune disease observed in $\mathrm{DH}$ patients with those in NOD mice, autoimmune thyroiditis is the most common, followed by Idd and lupus erythematosus (33). Ceat1, located in Idd16, has been identified as a gene critical for the development of experimental autoimmune thyroiditis in the NOD mouse (34). Determining which of these Idd loci contribute to the development of $\mathrm{DH}$ would require analyzing all the NOD Idd congenic mice in the context of an $\mathrm{Ab}^{\circ} \mathrm{DQ}^{+}$construct.

The exclusive appearance of blistering in these gluten-sensitive DQ8 transgenic NOD mice in the absence of enteropathy may be a consequence of the intraperitoneal route of sensitization as opposed to footpad injection. Although both celiac disease and $\mathrm{DH}$ have elements of combined cellular and humoral responses, $\mathrm{DH}$ is generally considered to be predominantly a Th2-mediated disease and celiac disease to be a Th1-mediated disease (35-37). The intraperitoneal injections, which typically generate humoral responses, may therefore be contributing to the development of a humoral disease that is skewed toward a Th2 response. We are currently comparing different combinations of injection routes to understand how the type of response to gluten (cellular vs. humoral, Th1 vs. Th2) will determine specific disease development.

Interestingly, no IgA antibodies specific for $\mathrm{tTG}$ or gliadin were detected in the sera of these mice. Also, indirect staining using sera from blistering mice did not bind to normal mouse skin or saltsplit mouse skin. Other studies have demonstrated that the sera from $\mathrm{DH}$ patients fail to bind to human skin, which is similar to the results in this mouse model $(20,38,39)$. However, it has been observed that $71 \%$ of $\mathrm{DH}$ patients had IgA reactivity specific for gliadin. This observation led to the hypothesis that an immune response against gliadin is initiated in the gut of DH patients, and circulating IgA/gliadin immune complexes deposit in the skin of affected individuals (40). However, no explanation was provided to account for the remaining third of $\mathrm{DH}$ patients who did not have circulating gliadin-specific IgA yet still developed glutendependent blistering. Further study of celiac disease revealed that patients on a normal diet had circulating IgA that was specific for tTG, an autoantigen that is likely exposed in damaged gut (41). Subsequent studies of DH patients on normal diets also found circulating antibodies against $\mathrm{tTG}$, with $70 \%$ of $\mathrm{DH}$ patients positive for IgA against tTG $(2,42,43)$. These findings reinforce the autoimmune nature of $\mathrm{DH}$, which is supported by the increased association of DH with other autoimmune diseases (32). Similarly, approximately $70 \%$ of $\mathrm{DH}$ patients have circulating IgA against reticulin and endomysium, both of which are diagnostic markers for celiac disease $(2,44)$. The recurring positivity of approximately $70 \%$ for anti-gliadin, anti-tTG, anti-reticulin, and anti-endomysium antibodies likely reflects the state of enteropathy in $\mathrm{DH}$ patients.

Sardy et al. reported in 2002 that a majority of DH patients harbor circulating IgA antibodies specific for TGe (30). Our inability to detect circulating IgA specific for TGe was unlikely related to the use of human TGe as the substrate, since there is $84 \%$ similarity in the amino acid sequence between human and mouse TGe (31). An antibody raised in rabbits against recombinant human TGe also stained mouse ear TGe, demonstrating cross-species reactivity (45). However, IgA deposits were observed to be in close proximity to the epidermis, where TGe may be exposed during the blistering process (Figure 6C).

Related to the difficulty of identifying the specificity of the IgA deposited beneath the dermal-epidermal junction is the challenge of determining the order of events that are required for the devel- 
opment of the blistering and related pathology observed in DH. IgA deposits have been found to be symptomatic in $\mathrm{DH}$ patients maintained on strict gluten-free diets for 10-13 years. We also observed IgA deposits in the ear skin of mice that had been treated for 5 weeks with a gluten-free diet and dapsone. This suggests that the presence of IgA deposits alone does not cause blistering.

One potential mechanism may involve neutrophils that infiltrate the dermis, bind to the IgA with FcoRI, and subsequently release granule enzymes that cause damage to the dermal-epidermal junction, resulting in the formation of blisters (46). Our finding of neutrophil elastase distributed within the epidermis would support this theory. The mediators that recruit and activate neutrophils within these sites of IgA deposition remain unknown (47).

This animal model of $\mathrm{DH}$ has provided novel information that suggests the following: (a) Blistering occurs only after a protracted period of gluten consumption between 2 and 5 months after gluten sensitization. (b) IgA deposition occurs beneath the basement membrane zone in the absence of enteropathy and circulating IgA specific for gliadin or tTG. (c) Neutrophils present in the dermis release neutrophil elastase that potentially contributes to the development of the subepidermal split and formation of the blister $(48,49)$. (d) Finally, significant numbers of $\mathrm{CD}^{+} \mathrm{T}$ cells need not be present within the skin for the development of blisters.

It is perplexing why a predominance of neutrophils and monocytes is drawn into the skin in the virtual absence of $\mathrm{CD}^{+}$ $\mathrm{T}$ cells. Like the perilesional skin of human $\mathrm{DH}$ patients, perilesional sections from blistered DH mice with IgA deposits contain few or no neutrophils or monocytes in the dermis. This would indicate that there are further events after IgA deposition to recruit the neutrophils, monocytes, and other inflammatory cells and mediators. Thus, though the CD4/DQ8 component of the immune reaction may be necessary for the generation of IgA deposits in the skin, it may not be necessary in the recruitment of pathogenic neutrophils and monocytes. Determining the specific mediators and cells involved in the engagement of neutrophils and monocytes and in their immigration to skin following ingestion of gluten, as well as understanding the exact role of the IgA deposits in the blistering process, is the focus of ongoing investigation using this novel mouse model of $\mathrm{DH}$.

\section{Methods}

Generation of transgenic mice. Transgenic mice that express HLA-DQ8 in an endogenous MHC II-deficient background $\left(\mathrm{Ab}^{\circ} \mathrm{DQ}^{+}\right)$were backcrossed to NOD mice for 10 generations and intercrossed to produce congenic NOD $\mathrm{Ab}^{\circ} \mathrm{DQ8}^{+}$mice (50).

Gluten sensitization. Mice 7-16 weeks of age, weaned on normal mouse chow (see below), were injected intraperitoneally with $50 \mathrm{ng}$ pertussis toxin (Sigma-Aldrich) in $100 \mu \mathrm{l}$ PBS. Three days later, they were injected intraperitoneally with $100 \mu \mathrm{g}$ crude gluten (Sigma-Aldrich) in $100 \mu \mathrm{l}$ CFA (Difco; BD). Twenty milligrams of crude gluten (Sigma-Aldrich) was administered twice weekly by gavage thereafter. These mice were monitored for blistering and weight loss twice weekly.

Photographic imaging. Digital images were captured using a FinePix 6800 Zoom Fujifilm digital camera (Fuji Photo Film USA Inc.).

Rodent chows. The chow designated as gluten-free was a purifiedingredient mouse chow obtained from Research Diets Inc. (rodent diet AIN-76A). It contains casein, methionine, cystine, corn starch, sucrose, maltodextrin, cellulose, corn oil, soybean oil, minerals, and vitamins. The chow designated as normal or regular mouse chow (LabDiet; PMI
Nutrition International) contains ground wheat, soybean meal, wheat germ meal, fish meal, animal fat preserved with butylated hydroxyanisole (BHA), dried whey, casein, soybean oil, calcium carbonate, corn gluten meal, salt, minerals, and vitamins.

Dapsone treatment. Forty micrograms of 4-aminophenyl sulfone (dapsone; Sigma-Aldrich) was administered daily by gavage for 4-6 weeks. The dosage is approximately $1 \mathrm{mg} / \mathrm{kg}$ daily with an average mouse weight of $30 \mathrm{~g}$, which is similar to human dosages based on a $\mathrm{mg} / \mathrm{kg}$ daily dose (26).

Ear biopsy. Biopsies of the ear were performed antemortem and postmortem. Antemortem biopsies consisted of anesthesia of the animal by isoflurane inhalation (Abbott Laboratories) and then use of a skin punch (Acuderm Inc.) to remove a small section of the ear. Postmortem biopsy sections were taken from the whole ear. Samples from lesional skin, perilesional uninvolved skin, or nonlesional (never-involved) skin were either snap-frozen in liquid nitrogen for direct or indirect immunofluorescent analysis or fixed in formalin mounted in paraffin sectioned and processed for $\mathrm{H} \& \mathrm{E}$ staining.

Small-intestine analysis. After sacrifice, the intestines were removed, and 1$\mathrm{cm}$-long sections of the ileum, jejunum, and duodenum were removed and flushed with calcium and magnesium free (CMF; $2 \%$ FBS, $1 \times$ HEPES, $1 \times$ HBSS). The $1-\mathrm{cm}$ sections were then further dissected, such that 2 vertical sections of $2 \mathrm{~mm}$ depth and 1 longitudinal section of $4 \mathrm{~mm}$ length sliced longitudinally were placed into formalin.

$H \mathcal{E} E$ staining. The formalin-fixed skin and intestinal specimens were paraffin-embedded, sectioned, processed, and stained with H\&E.

Direct immunofluorescence testing. Five-micron-thick cryostat (Leica CM3050; Leica Microsystems Inc.) sections from each specimen were placed on frosted glass slides (Ultra Frost Rite-on; BD). FITC-conjugated anti-mouse IgA (1:4 dilution; $1.1 \mathrm{mg} / \mathrm{ml}$; Sigma-Aldrich) or FITC-conjugated anti-mouse IgG (1:300 dilution; $16 \mathrm{~g} / \mathrm{ml})$ was used to detect mouse IgA or IgG deposits within the sections. Purified anti-mouse collagen IV was purchased from USBiological Inc. and detected using Rhodamine Red-X-conjugated anti-rabbit IgG from Jackson ImmunoResearch Laboratories Inc. Purified anti-recombinant human TGe antibody was kindly provided by Soo Youl Kim and detected using Rhodamine Red-X-conjugated anti-rabbit IgG. FITC-conjugated antineutrophil antibody $7 / 4$ was purchased from Caltag Laboratories Inc. FITC-conjugated anti-keyhole limpet hemocyanin (anti-KLH) rat IgG was used as the isotype control for antibody 7/4 (BD Biosciences - Pharmingen). Purified anti-neutrophil elastase was purchased from Santa Cruz Biotechnology Inc. Whole goat IgG was used as the isotype control for anti-neutrophil elastase (Jackson ImmunoResearch Laboratories Inc.). Purified anti-mouse MBP antibody (14.7.4) was kindly provided by James Lee (Mayo Clinic, Scottsdale, Arizona, USA). FITCconjugated anti-CD4 and anti-CD8 as well as purified anti-c-kit were obtained from BD Biosciences - Pharmingen. Secondary antibodies were obtained from Jackson ImmunoResearch Laboratories Inc. and consisted of FITC-conjugated anti-goat IgG for anti-neutrophil elastase and FITC-conjugated anti-rat IgG for 14.7.4, and anti-c-kit. Slides were viewed using 3 different microscopes, a Zeiss Axioplan microscope (Carl Zeiss International), an Olympus AX70 Research Microscope (Olympus Corp.), and a Zeiss Axiovert 100M confocal laser scanning microscope (Carl Zeiss International).

Indirect staining using mouse and human skin. Ear biopsies from naive NOD $\mathrm{Ab}^{\circ} \mathrm{DQ}^{+}$mice and control human skin biopsies were placed into $1.0 \mathrm{M}$ $\mathrm{NaCl}$ and incubated at $4{ }^{\circ} \mathrm{C}$ for 2-3 days. Once blisters appeared on the skin, the samples were snap-frozen in liquid nitrogen and processed as described in the preceding section. Sera were diluted 1:20 for staining, and FITC-conjugated anti-IgA and FITC-conjugated anti-IgG were used to detect skin-bound sera. 
Detection of endomysial antibodies. Sera from blistered mice were added at a dilution of 1:20 to monkey esophagus (Oregon National Primate Research Center), and secondary antibodies were anti-mouse IgA (1:4) or anti-mouse IgG (1:300).

Anti-tTg and-TGe ELISA. Tissue transglutaminase was derived from guinea pigs (Sigma-Aldrich). Recombinant human epidermal transglutaminase was kindly provided by the late Peter Steinert. Transglutaminase was resuspended in PBS at a concentration of $1 \mu \mathrm{g} / \mathrm{ml}$ and coated overnight at $4^{\circ} \mathrm{C}$ onto Immulon 2 plates (Fisher Scientific International Inc.). Plates were blocked with Tris-buffered saline (TBS) plus 0.05\% Tween-20 (SigmaAldrich) for 1 hour at room temperature. Sera were diluted 1:100 and 1:200 in TBS for anti-TGe and 1:200 and 1:400 for anti-tTG and incubated for 1 hour at room temperature. Secondary antibody was either biotinylated rat anti-mouse IgA $(1: 1,000)$ from Accurate Chemical \& Scientific Corp., or biotinylated anti-mouse IgG $(1: 1,000)$ from Jackson ImmunoResearch Laboratories Inc. The enzyme conjugate was streptavidin-HRP from Jackson ImmunoResearch Laboratories Inc., and the substrate was 3,3',5,5'tetramethylbenzidine (TMB) from Sigma-Aldrich.

Anti-gliadin and-gluten ELISAs. Crude gliadin or gluten (Sigma-Aldrich) was resuspended in methanol at $10 \mathrm{mg} / \mathrm{ml}$, and then diluted into absolute ethanol at a concentration of $1 \mu \mathrm{g} / \mathrm{ml}$. One hundred microliters of the $1 \mu \mathrm{g} / \mathrm{ml}$ gliadin/gluten ethanol solution was placed into each well of an Immulon 2 plate (Fisher Scientific International Inc.) and then allowed to dry under a hood. The plate was then blocked with $4 \%$ BSA/PBS for 2 hours at $37^{\circ} \mathrm{C}$. The plate was washed with $1 \times$ PBS, $0.05 \%$ Tween-20. Sample sera were diluted into $0.1 \%$ BSA/PBS 1:200, 1:400, and 1:800 and incubated for 1 hour at $37^{\circ} \mathrm{C}$. Detection antibodies were biotinylated rat anti-mouse IgA from Accurate Chemical \& Scientific Corp., and biotinylated anti-mouse IgG from Jackson ImmunoResearch
Laboratories Inc. The enzyme conjugate was streptavidin-HRP, and the substrate was TMB.

Total IgA and IgG ELISAs. Sample sera were diluted into PBS 1:200, 1:400, and 1:800 for IgA and 1:400, 1:800, and 1:1,600 for IgG analysis. Plates were coated overnight at $4^{\circ} \mathrm{C}$ and blocked with $4 \%$ BSA/PBS for 1 hour at $37^{\circ} \mathrm{C}$. Detection antibodies were rat anti-mouse IgA (Accurate Chemical \& Scientific Corp.) and anti-mouse IgG (Jackson ImmunoResearch Laboratories Inc.). Plates were incubated for 1 hour at $37^{\circ} \mathrm{C}$. The enzyme conjugate was streptavidin-HRP, and the substrate was TMB.

Glucose measurement. A DEX glucometer (Bayer Corp.) was used to test the glucose level in the blood of mice. Glucose measurements were performed once every 2 months after the development of blistering, using blood droplets that were extracted by tail clipping.

These studies were approved by the Mayo Foundation Institutional Animal Care and Use Committee.

\section{Acknowledgments}

This work was supported by grants from the Oberkotter Foundation, NIH grants AI14764 and DK57892, and the Mayo Foundation. We would also like to acknowledge the help of Soo Youl Kim and the late Peter Steinert in providing the TGe reagents.

Received for publication January 13, 2004, and accepted in revised form August 24, 2004.

Address correspondence to: Joseph A. Murray, Department of Gastroenterology and Hepatology, Mayo Foundation, Rochester, Minnesota 55905, USA. Phone: (507) 284-2631; Fax: (507) 266-9081; E-mail: murray.joseph@mayo.edu.
1. Jordan, R.E. 2000. Dermatitis herpetiformis. In Atlas of bullous disease. L. Fathman, editor. Churchill Livingstone. Philadelphia, Pennsylvania, USA. 99-107.

2. Fry, L. 2002. Dermatitis herpetiformis: problems, progress and prospects. Eur. J. Dermatol. 12:523-531.

3. Lever, W.F., and Schaumburg-Lever, G. 1983. Noninfectious vesicular and bullous diseases. In Histopathology of the skin. 6 th edition. J.B. Lippincott Co. Philadelphia, Pennsylvania, USA. 92-136.

4. Fry, L. 1995. Dermatitis herpetiformis. Baillieres Clin. Gastroenterol. 9:371-393.

5. Garioch, J.J., Lewis, H.M., Sargent, S.A., Leonard, J.N., and Fry, L. 1994. 25 years' experience of a gluten-free diet in the treatment of dermatitis herpetiformis. Br. J. Dermatol. 131:541-545.

6. Reunala, T. 1998. Dermatitis herpetiformis: coeliac disease of the skin. Ann. Med. 30:416-418.

7. Spurkland, A., et al. 1997. Dermatitis herpetiformis and celiac disease are both primarily associated with the HLA-DQ (alpha $1 * 0501$, beta $1 * 02$ ) or the HLA-DQ (alpha $1 * 03$, beta $1 * 0302$ ) heterodimers. Tissue Antigens. 49:29-34.

8. Hervonen, K., et al. 2000. Concordance of dermatitis herpetiformis and celiac disease in monozygous twins. J. Invest. Dermatol. 115:990-993.

9. Zhu, Y.I., and Stiller, M.J. 2001. Dapsone and sulfones in dermatology: overview and update. J. Am. Acad. Dermatol. 45:420-434.

10. Miyachi, Y. 2000. Pharmacologic modulation of neutrophil functions. Clin. Dermatol. 18:369-373.

11. Mobacken, H., Andersson, H., Dahlberg, E., and Kastrup, W. 1987. Relationship of dietary gluten intake to dapsone dose in dermatitis herpetiformis. Acta Derm. Venereol. 67:267-270.

12. Marsella, R., and Olivry, T. 2003. Animal models of atopic dermatitis. Clin. Dermatol. 21:122-133.

13. Matsumoto, M., et al. 1999. IgE hyperproduction through enhanced tyrosine phosphorylation of Janus kinase 3 in NC/Nga mice, a model for human atopic dermatitis. J. Immunol. 162:1056-1063.

14. HogenEsch, H., Janke, S., Boggess, D., and Sundberg, J.P. 1999. Absence of Peyer's patches and abnormal lymphoid architecture in chronic proliferative dermatitis (cpdm/cpdm) mice. J. Immunol. 162:3890-3896.

15. Schon, M., Behmenburg, C., Denzer, D., and Schon, M.P. 2001. Pathogenic function of IL-1 beta in psoriasiform skin lesions of flaky skin (fsn/fsn) mice. Clin. Exp. Immunol. 123:505-510.

16. Hikita, I., et al. 2002. Characterization of dermatitis arising spontaneously in DS-Nh mice maintained under conventional conditions: another possible model for atopic dermatitis. J. Dermatol. Sci. 30:142-153.

17. Saint-Mezard, P., et al. 2003. Afferent and efferent phases of allergic contact dermatitis (ACD) can be induced after a single skin contact with haptens: evidence using a mouse model of primary ACD. J. Invest. Dermatol. 120:641-647.

18. Li, X.M., et al. 2001. Murine model of atopic dermatitis associated with food hypersensitivity. J. Allergy Clin. Immunol. 107:693-702.

19. Gijbels, M.J., et al. 2000. Therapeutic interventions in mice with chronic proliferative dermatitis (cpdm/cpdm). Exp. Dermatol. 9:351-358.

20. Zone, J.J., Egan, C.A., Taylor, T.B., and Meyer, L.J. 2004. IgA autoimmune disorders: development of a passive transfer mouse model. J. Investig. Dermatol. Symp. Proc. 9:47-51.

21. Hall, E.J., and Batt, R.M. 1992. Dietary modulation of gluten sensitivity in a naturally occurring enteropathy of Irish setter dogs. Gut. 33:198-205.

22. Black, K.E., Murray, J.A., and David, C.S. 2002. HLADQ determines the response to exogenous wheat proteins: a model of gluten sensitivity in transgenic knockout mice. J. Immunol. 169:5595-5600.

23. Johansson, A.C., Lindqvist, A.K., Johannesson, M., and Holmdahl, R. 2003. Genetic heterogeneity of autoimmune disorders in the nonobese diabetic mouse. Scand. J. Immunol. 57:203-213.

24. Hou, W., et al. 2003. Pertussis toxin enhances Th 1 responses by stimulation of dendritic cells. J. Immunol. 170:1728-1736.

25. Ryan, M., McCarthy, L., Rappuoli, R., Mahon, B.P., and Mills, K.H. 1998. Pertussis toxin potentiates Th1 and Th 2 responses to co-injected antigen: adjuvant action is associated with enhanced regulatory cytokine production and expression of the co-stimulatory molecules B7-1, B7-2 and CD28. Int. Immunol. 10:651-662.

26. Zone, J.J., Meyer, L.J., and Petersen, M.J. 1996. Deposition of granular IgA relative to clinical lesions in dermatitis herpetiformis. Arch. Dermatol. 132:912-918.

27. Otley, C., and Hall, R.P., 3rd. 1990. Dermatitis herpetiformis. Dermatol. Clin. 8:759-769.

28. Haffenden, G., Wojnarowska, F., and Fry, L. 1979. Comparison of immunoglobulin and complement deposition in multiple biopsies from the uninvolved skin in dermatitis herpetiformis. Br.J. Dermatol. 101:39-45.

29. Gentile, V., et al. 1991. Isolation and characterization of cDNA clones to mouse macrophage and human endothelial cell tissue transglutaminases. J. Biol. Chem. 266:478-483.

30. Sardy, M., Karpati, S., Merkl, B., Paulsson, M., and Smyth, N. 2002. Epidermal transglutaminase (TGase 3) is the autoantigen of dermatitis herpetiformis. J. Exp. Med. 195:747-757.

31. Kim, I.G., Gorman, J.J., Park, S.C., Chung, S.I., and Steinert, P.M. 1993. The deduced sequence of the novel protransglutaminase E (TGase 3 ) of human and mouse. J. Biol. Chem. 268:12682-12690.

32. Gaspari, A.A., et al. 1990. Prevalence of thyroid abnormalities in patients with dermatitis herpetiformis and in control subjects with HLA-B8/-DR3. Am. J. Med. 88:145-150.

33. Reunala, T., and Collin, P. 1997. Diseases associ- 
ated with dermatitis herpetiformis. Br. J. Dermatol. 136:315-318.

34. Boulard, O., et al. 2002. An interval tightly linked to but distinct from the $\mathrm{H} 2$ complex controls both overt diabetes (Idd16) and chronic experimental autoimmune thyroiditis (Ceat 1 ) in nonobese diabetic mice. Diabetes. 51:2141-2147.

35. Caproni, M., et al. 1998. Th2-like cytokine activity in dermatitis herpetiformis. Br. J. Dermatol. 138:242-247.

36. Salvati, V.M., et al. 2002. Interleukin 18 and associated markers of $\mathrm{T}$ helper cell type 1 activity in coeliac disease. Gut. 50:186-190.

37. Smith, A.D., Bagheri, B., Streilein, R.D., and Hall, R.P., 3rd. 1999. Expression of interleukin-4 and interferon-gamma in the small bowel of patients with dermatitis herpetiformis and isolated glutensensitive enteropathy. Dig. Dis. Sci. 44:2124-2132.

38. Hall, R.P., and Lawley, T.J. 1985. Characterization of circulating and cutaneous IgA immune complexes in patients with dermatitis herpetiformis. J. Immunol. 135:1760-1765.

39. Wakelin, S.H., and Wojnarowska, F. 1997. Immu- nobullous diseases. In Skin immune system (SIS): cutaneous immunology and clinical immunodermatology. 2nd edition. J.D. Bos, editor. CRC Press. Boca Raton, Florida, USA. 445-460.

40. Vainio, E., Kalimo, K., Reunala, T., Viander, M., and Palosuo, T. 1983. Circulating IgA- and IgG-class antigliadin antibodies in dermatitis herpetiformis detected by enzyme-linked immunosorbent assay. Arch. Dermatol. Res. 275:15-18.

41. Dieterich, W., et al. 1997. Identification of tissue transglutaminase as the autoantigen of celiac disease. Nat. Med. 3:797-801.

42. Porter, W.M., et al. 1999. Tissue transglutaminase antibodies in dermatitis herpetiformis. Gastroenterology. 117:749-750.

43. Dieterich, W., et al. 1999. Antibodies to tissue transglutaminase as serologic markers in patients with dermatitis herpetiformis. J. Invest. Dermatol. 113:133-136.

44. Reunala, T., et al. 1987. IgA anti-endomysial antibodies in dermatitis herpetiformis: correlation with jejunal morphology, gluten-free diet and antigliadin antibodies. Br. J. Dermatol. 117:185-191.
45. Candi, E., et al. 2002. Expression of transglutaminase 5 in normal and pathologic human epidermis. J. Invest. Dermatol. 119:670-677.

46. Graeber, M., et al. 1993. The role of cytokines in the generation of skin lesions in dermatitis herpetiformis. Br. J. Dermatol. 129:530-532.

47. Smith, A.D., Streilein, R.D., and Hall, R.P., 3rd. 2002. Neutrophil CD11b, L-selectin and Fc IgA receptors in patients with dermatitis herpetiformis. Br. J. Dermatol. 147:1109-1117.

48. Oikarinen, A.I., Zone, J.J., Ahmed, A.R., Kiistala, U., and Uitto, J. 1983. Demonstration of collagenase and elastase activities in the blister fluids from bullous skin diseases. Comparison between dermatitis herpetiformis and bullous pemphigoid. J. Invest. Dermatol. 81:261-266.

49. Liu, Z., et al. 2000. A critical role for neutrophil elastase in experimental bullous pemphigoid. J. Clin. Invest. 105:113-123.

50. Kudva, Y.C., et al. 2002. Modulation of insulitis and type 1 diabetes by transgenic HLA-DR3 and DQ8 in NOD mice lacking endogenous MHC class II. Hum. Immunol. 63:987-999. 